\section{Genetic variations can point the way to disease genes}

\section{London}

Researchers have shown for the first time that the search for human genes can be substantially narrowed using maps of small variations in the genome, according to a presentation this week at the American Society of Human Genetics (ASHG). They also claim that the density of variations needed is much lower than some have suggested.

The search for disease genes usually involves long sections of DNA, as traditional markers are widely spaced. An alternative is to use maps of single nucleotide polymorphisms (SNPs), based on the difference between individuals. Because SNPs are usually either part of a gene or close to a gene, they are seen as more effective than conventional methods for locating disease genes.

But some researchers have argued that a high density of SNPs would be needed to detect association between a marker and a disease. A study earlier this year based on simulations suggested that one SNP per 6,000 base pairs was needed - a total of 500,000 for whole-genome studies (Nature Genetics 22, 139-144; 1999). If true, says Allen Roses, director of genetics at Glaxo Wellcome, this would "put a damper" on the idea of researchers using SNPs because the technique is "tremendously expensive".

But Roses claims that his work on other diseases has shown that an SNP map with a density of one per 10,000 30,000 base pairs could be used to identify areas of DNA thought to be linked to a disease - five times less dense than previously thought.

Roses told the ASHG at its meeting in San Francisco this week that Glaxo Wellcome scientists have already used SNPs to locate areas of DNA for three diseases: migraine; type II diabetes; and psoriasis. Roses also says he has shown that, given a known susceptibility gene, APOE in Alzheimer's disease, SNPs can narrow its location down from a strip of DNA four million base pairs in length to only 40,000 base pairs - a region containing only two genes.

"It is important to demonstrate that it is possible to narrow the region by orders of magnitude," says Roses. "Nobody has done SNP mapping before because it is tremendously expensive and unproven. We felt it important to demonstrate to the academic world that it is possible." Natasha Loder

\title{
NASA plans to map stars and hunt gamma-ray bursts ...
}

Washington

The US space agency NASA last week gave the go-ahead to two new medium-class Explorer spacecraft in the next five years. One will study gamma rays, and the second will map 40 million stars with unprecedented precision.

The \$163-million Swift Gamma-ray Burst Explorer, scheduled to reach orbit in 2003, will have three telescopes - viewing in gamma-ray, X-ray and ultraviolet/visible wavelengths - poised to observe these mysterious bursts within minutes of their appearance.

During its three-year mission, Swift is expected to pinpoint some 300 gamma-ray bursts, and to discover about 400 black holes. The principal investigator is Neil Gehrels of NASA's Goddard Space Flight Center in Maryland.

The \$162-million Full-sky Astrometric Mapping Explorer (FAME), due to launch in 2004 , is intended to improve on the successful European Hipparcos mission of the early 1990s, which gave extremely accurate positions (to within 1 milliarcsecond) for 120,000 stars.
FAME will use advanced charge-coupled device arrays and a more efficient scheme for blocking out sunlight to pinpoint some 40 million stars down to fifteenth magnitude, with an accuracy of 500 microarcseconds. For stars brighter than ninth magnitude the accuracy will be 50 microarcseconds.

"There's all kinds of science you can do with these observations," says Kenneth Seidelmann, leader of the project's science working team at the US Naval Observatory in Washington DC.

A key objective is the search for wobbles in a star's motion that betray the presence of a large planet or brown dwarf. Seidelmann says FAME should be able to detect planets that are as small as twice the mass of Jupiter.

During its five-year, all-sky survey FAME will produce a catalogue of star locations that can be used by NASA's planned followon, the Space Interferometry Mission, which will be accurate to one microarcsecond. The European Space Agency is also considering an astrometry mission called GAIA, which could catalogue a billion objects with similar precision.

Tony Reichhardt

\section{... and Europe to measure the Earth's gravity}

Munich

A major mission to determine the Earth's gravity field at high resolution has been recommended by Earth scientists as top priority in the European Space Agency's (ESA's) Earth Observation programme, 'Living Planet'.

The mission, called GOCE (Gravity Field and Steady State Ocean Circulation Explorer), was selected by ESA's Earth Sciences Advisory Committee in a competition held in Granada last week between four proposals developed by the European scientific community.

An Atmospheric Dynamics Mission (ADM) - a laser system for measuring wind and wind profiles from space - was the runner-up in the competition.

Both missions are expected to be approved by ESA's decision-making

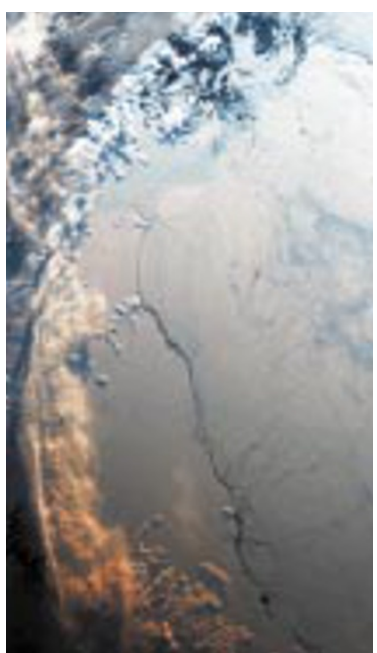

Up and away: Antarctica's Larsen Ice Shelf from space.

Science Programme

Committee next month.

They should also

provide important data for climate research.

GOCE will give information on irregularities in the mass distribution of the Earth by measuring variations in the gravity field.

This, together with satellite altimetry of the oceans' surface, will enable oceanographers to determine ocean circulation in more detail. They will also be able to assess the variation in the polar ice caps more accurately.

By measuring winds at different heights, ADM will help to clarify how the atmosphere moves. GOCE will also

improve estimates of the density of the Earth's lithosphere and upper mantle.

GOCE is set for a launch in mid-2004, and ADM in 2006. Both missions will cost less than 400 million euros (US\$435 million) and will be run by scientific teams consisting of members from most of the ESA's member states.

Alison Abbott 